\title{
De nouvelles technologies au service du maintien à domicile des personnes âgées
}

BRUNO PAIS ${ }^{\mathrm{a}}$, Dr PHILIPP BULUSCHEK ${ }^{\mathrm{b}}$, Pr TOBIAS NEF ${ }^{\mathrm{c}}$, NARAYAN SCHÜTZ ${ }^{\mathrm{c}}$, Pr HUGO SANER ${ }^{\mathrm{c}, \mathrm{d}}$, Prs DANIEL GATICA ${ }^{\mathrm{e}}$ et VALÉRIE SANTSCHI ${ }^{\mathrm{a}}$

Rev Med Suisse 2019; 15: 1407-11

\begin{abstract}
Le vieillissement de la population suisse augmente les besoins et les coûts liés à la santé. La fragilité et les maladies chroniques touchant les personnes âgées diminuent leur capacité à vivre de façon autonome. Cependant, la grande majorité d'entre eux souhaitent continuer à vivre chez eux, tout en ayant une qualité de vie et en recevant les meilleures prestations de soins. Dans ce contexte, de nouvelles technologies de santé connectée peuvent être une solution pertinente pour faciliter le maintien à domicile des personnes âgées. Nous présentons, dans cet article, les enjeux en lien avec ces technologies et, plus particulièrement, dans quelle mesure pourraient-elles contribuer au maintien à domicile des personnes âgées et être une plus-value pour les patients et les proches, mais aussi pour les médecins et les autres professionnels de santé. Enfin, les craintes et les risques associés à ces technologies et l'importance d'évaluer scientifiquement leur utilité sont discutés.
\end{abstract}

\section{New healthcare technologies to support home care of older people}

The ageing of the Swiss population is increasing the healthcare needs and costs. Both frailty and chronic diseases affecting older people reduce their ability to live independently. However, the vast majority of older people want to continue living at home, while having a quality of life and receiving the best healthcare services. In this context, new connected healthcare technologies can be a relevant solution to facilitate home care of older people. In this article, we present the issues related to these technologies and, more particularly, to what extent they could contribute to home care of older people and be a benefit for patients and family caregivers, but also for physicians and other healthcare professionals. Finally, the fears and risks associated with these technologies, and the importance of scientifically assessing their usefulness are discussed.

\section{INTRODUCTION}

La Suisse est confrontée à un vieillissement progressif de sa population en raison de l'augmentation de l'espérance de vie et d'une natalité relativement faible. Par exemple, les personnes âgées de plus de 65 ans représentent aujourd'hui plus

a Institut et Haute Ecole de la Santé La Source, HES-SO Haute école spécialisée

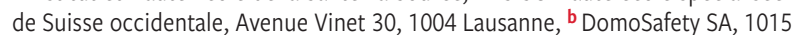
Lausanne, ' $A$ ARTORG Center for Biomedical Engineering Research, Université de Berne, 3012 Berne, d Hôpital de l'lle, 3010 Berne, e Idiap Research Institute, 1920 Martigny

v.santschi@ecolelasource.ch de $18 \%$ de la population contre $10 \%$ en 1990 . Selon les projections de l'Office fédéral de la statistique (OFS), le vieillissement de la population va s'accentuer, et les personnes de plus de 65 ans constitueront $27 \%$ de la population suisse en $2050 .{ }^{1}$ Cette évolution démographique a pour effet une augmentation du nombre de personnes avec des maladies chroniques, une augmentation du nombre de patients qui font appel au système de santé, et une augmentation des coûts liés à la santé. ${ }^{2}$ Selon une récente étude, $79 \%$ des coûts de la santé en Suisse seraient liés à la prise en charge des maladies non transmissibles chroniques. ${ }^{3,4} \mathrm{Au}$ vu de l'augmentation du nombre de personnes âgées, le fardeau des maladies chroniques et de leurs conséquences qui s'accroît nécessite d'adapter le système de santé et la prise en charge des personnes âgées qui manifestent le souhait de vivre le plus longtemps possible à domicile. ${ }^{5,6}$

Dans ce contexte, les technologies de l'information et de la communication, et plus particulièrement la santé connectée, peuvent offrir un potentiel intéressant pour améliorer la gestion des maladies chroniques et répondre au souhait des personnes âgées de vivre le plus longtemps possible à domicile. La santé connectée pourrait aussi faciliter la coordination des soins et favoriser l'autonomie et le maintien des personnes âgées au domicile en les accompagnant à devenir acteurs de leur santé.

\section{SANTÉ CONNECTÉE: DE QUOI PARLE-T-ON?}

La santé connectée, e-santé (eHealth en anglais), ou santé numérique, désigne l'ensemble des nouvelles technologies de l'information et de la communication appliquées au domaine de la santé et qui participent à améliorer la prise en charge des patients et la qualité des soins, ${ }^{7,8}$ en facilitant le partage des données via des outils digitaux et en augmentant la disponibilité d'accès à ces informations au patient, aux médecins et aux autres professionnels de santé. La santé connectée s'appuie ainsi sur une plus grande participation du patient dans la gestion de sa santé et une meilleure mise en réseau avec les différents acteurs de soins et équipes de soins concernés.

$\mathrm{Au}$ sein du domaine de la santé connectée, on distingue la $\mathrm{m}$-santé qui couvre la santé mobile via les applications santé sur smartphone et les objets connectés (bracelets d'activité, balances intelligentes, appareils de mesure de pression artérielle) qui mesurent, enregistrent et analysent, par exemple, les paramètres physiologiques du patient en lien avec sa santé. La santé connectée recouvre aussi le dossier électronique du patient et la télémédecine. 
Plus récemment, s'est développé le champ de la domotique qui regroupe un ensemble de technologies consistant à assurer des fonctions de sécurité (avec des capteurs et des alarmes), de confort (ouverture des portes) ou de gestion d'énergie (programmation de chauffage) pour rendre l'habitat intelligent..$^{9-11}$ Par exemple, les technologies développées, dans le contexte du maintien à domicile, sont des capteurs analysant des signes vitaux et des paramètres environnementaux de façon à apporter une sécurité et un confort aux personnes âgées. ${ }^{12,13}$

\section{SANTÉ CONNECTÉE: QUELS BÉNÉFICES ET RISQUES POUR LE PATIENT ET LES PROFESSIONNELS DE SANTÉ?}

Près de $15 \%$ de la population suisse reçoivent une aide de la part de leurs proches ou bénéficient des prestations d'aide et de soins à domicile, et $59 \%$ de ces bénéficiaires souffrent de maladies chroniques limitant leurs activités quotidiennes. ${ }^{14}$ De plus, le maintien à domicile est un souhait souvent exprimé par les personnes âgées en perte d'autonomie, nécessitant ainsi un soutien et une prise en charge à domicile. Pour répondre à leurs besoins, des nouvelles technologies sont actuellement en développement et doivent satisfaire aux différentes attentes des patients, familles et professionnels de santé, en particulier sur les aspects de la sécurité, le confort, le respect de la vie privée, la prévention de la perte de mobilité et problèmes de santé, la détection des urgences vitales, ainsi que le maintien des relations sociales. ${ }^{15,16}$

Introduire de nouvelles technologies peut offrir de nouvelles perspectives de prise en charge des maladies chroniques, par exemple en intervenant rapidement auprès des personnes âgées lors de chutes ou d'accidents domestiques et permettre aussi une meilleure coordination des soins.

Néanmoins, l'introduction et l'utilisation des technologies de la santé dans la pratique clinique soulève aussi des craintes de la part des patients, de leur famille, des médecins et des autres professionnels de santé. ${ }^{17,18}$ Ainsi, les personnes âgées peuvent rapporter crainte, stress et réticence. Par exemple, l'utilisation de capteurs de mouvement fixés sur les murs ou les portes de son domicile, peut être considérée par la personne âgée comme encombrant, gênant, voire stressant et intrusif. Ils sont aussi parfois soucieux de la protection de leurs données personnelles. Néanmoins, s'ils sont bien informés, ils semblent moins inquiets de l'emploi de ces technologies.

Par ailleurs, les médecins et les professionnels de santé peuvent s'inquiéter de la potentielle perte de contact humain avec leur patient, il y aurait un risque de déshumanisation de la relation de soin. ${ }^{19}$ Les professionnels de santé se sentent parfois bousculés dans leurs habitudes professionnelles ou menacés par l'évolution rapide du métier, notamment dans la pratique des soins à domicile. Ils ne désirent pas être substitués par des technologies et s'inquiètent que le lien de confiance construit avec le patient soit altéré. Il est dès lors important de développer et tester ces nouveaux outils technologiques en collaboration avec les professionnels de santé, afin de répondre au mieux à leurs besoins. Les réticences ou contraintes ressenties par les personnes âgées sont souvent dissipées par l'aisance et le rapport que le personnel soignant développe et entretient avec ces nouvelles technologies.

La formation des soignants aux nouvelles technologies et l'information qui leur est transmise sur leur utilité et leurs bénéfices sont essentielles au succès du déploiement de ces technologies dans le cadre, par exemple, des soins à domicile ainsi qu'à l'adhésion des personnes âgées et des professionnels de santé..$^{20,21}$

\section{QUELLES SOLUTIONS TECHNOLOGIQUES POUR AIDER AU MAINTIEN À DOMICILE DES PERSONNES ÂGÉES?}

De nouvelles technologies de santé connectée voient le jour pour répondre aux besoins des personnes âgées dans leur vie quotidienne et les maintenir à domicile. Les objets connectés tels que les capteurs de mouvement, bracelets d'activité, montre d'alarme (figure 1) permettent, par exemple, de mesurer et d'analyser les activités quotidiennes (mobilité, utilisation du réfrigérateur, utilisation de la salle de bain, par exemple) ainsi que les paramètres physiologiques (cycles du sommeil, fréquence cardiaque, par exemple) des personnes

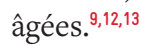

La revue de littérature de Peetoom et coll. ${ }^{22}$ regroupe des études menées majoritairement aux Etats-Unis évaluant l'utilisation des nouvelles technologies telles que les capteurs de mouvement, les bracelets d'activité ou les capteurs de lit, a montré que ces technologies sont potentiellement utiles au maintien à domicile des personnes âgées, en particulier pour détecter des modifications des habitudes de vie ou d'incident (chute, malaise). A ce jour, aucune étude n'a clairement démontré les éventuels effets positifs (meilleure autonomie et qualité de vie de la personne âgée) ainsi que les potentielles plus-values (prévention des problèmes de santé liés aux chutes, réduction des coûts liés au nombre et à la durée des hospitalisations) de ces nouvelles technologies. Les potentiels bénéfices de ces nouvelles technologies tels qu'une optimisation de l'organisation des soins à domicile, un meilleur suivi du patient par les professionnels de santé restent donc à démontrer dans la pratique clinique auprès des personnes âgées et des professionnels de santé..$^{21,22}$

En Suisse romande, l'étude SWISKO, menée par l'entreprise DomoSafety issue de l'EPFL, la Haute Ecole de la Santé La Source (HES-SO), les soins à domicile de NOMAD de Neuchâtel, l'Hôpital de l'Ile de Berne et son service de cardiologie, l'Idiap Research Institute de Martigny et le centre ARTORG de recherche biomédicale de l'Université de Berne, vise à évaluer une nouvelle solution technologique de domotique, pour favoriser le maintien à domicile. Ce projet, financé par la Commission pour la technologie et l'innovation de la Confédération (CTI), a pour objectif d'évaluer dans quelle mesure un nouvel outil technologique combinant des capteurs environnementaux et des capteurs portables peut être utile dans le maintien à domicile des personnes âgées (figure 2).

Pour ce faire, 25 personnes âgées de 75 ans et plus, vivant seules à domicile, sont suivies durant 12 mois par le personnel soignant des soins à domicile de NOMAD de Neuchâtel. La 


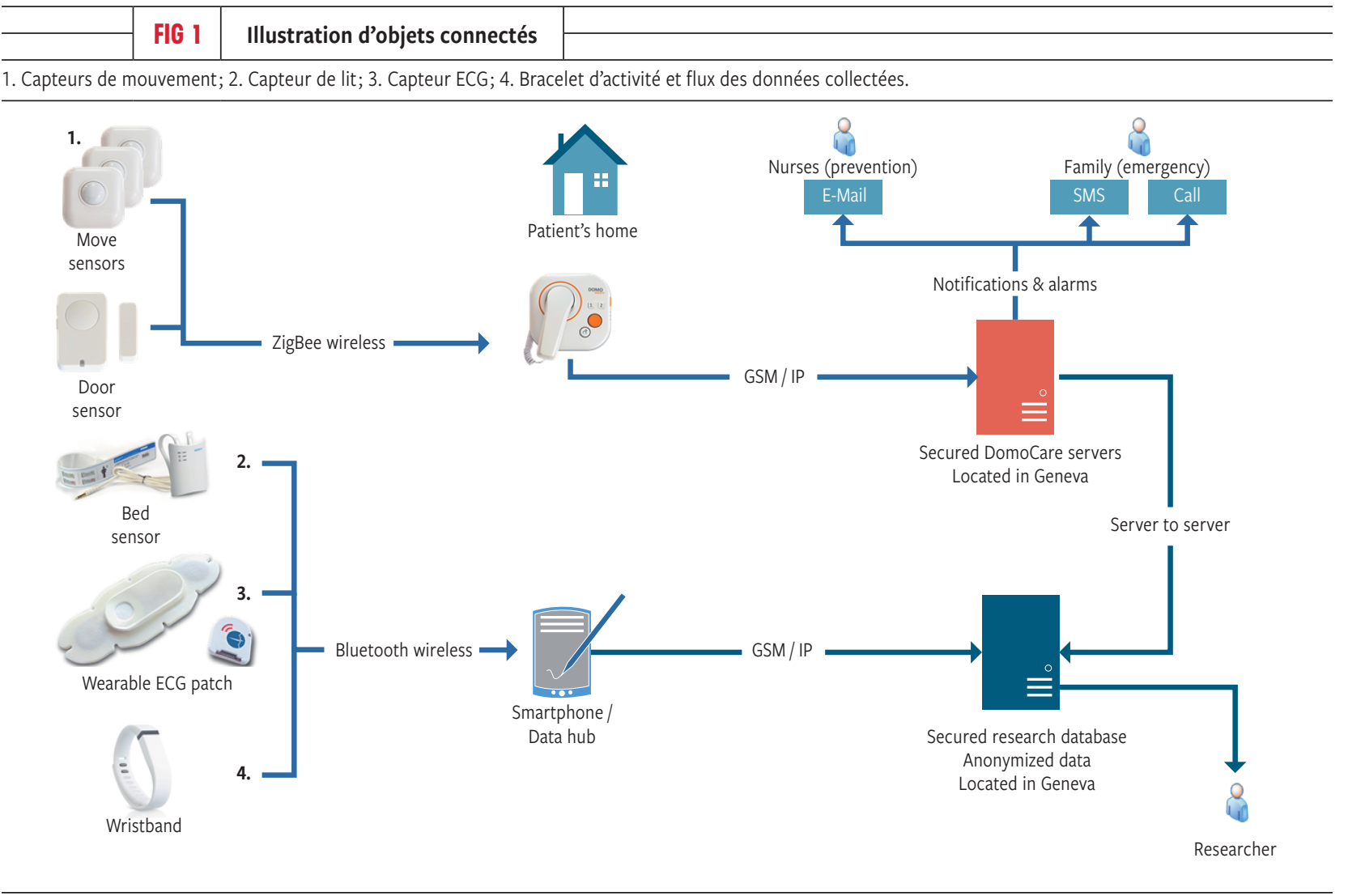

personne âgée qui accepte de participer à l'étude voit son appartement équipé de capteurs environnementaux de mouvement et de présence (lit), ainsi que de capteurs d'ouverture de porte permettant le suivi de ses activités quotidiennes. Les capteurs présents dans la maison envoient des informations sur les comportements et les habitudes de la personne âgée. En cas de modifications des habitudes de vie de la personne âgée ou d'un incident (chute, malaise), un message est envoyé aux infirmières de NOMAD qui peuvent agir en conséquence. La personne âgée qui a accepté de participer porte également durant l'étude un capteur portable au poignet sous la forme d'un bracelet permettant le suivi de son activité physique en nombre de pas, ainsi qu'un capteur portable au torse (fixé par un patch) mesurant son activité cardiaque.

Cette technologie de santé connectée permet un suivi, un échange d'informations entre le médecin et le professionnel de santé ainsi qu'une intervention du personnel soignant en cas de chute ou de pathologie plus grave. Par ailleurs, dans le cadre de cette étude, il est prévu d'évaluer l'avis des professionnels de santé et des patients quant à l'emploi et l'utilité de ces technologies.

Les résultats de ce projet sont attendus pour cette année. Ce projet est aussi un exemple de collaboration entre le milieu de la santé et le milieu universitaire et industriel, alliant des connaissances et des compétences multidisciplinaires pertinentes dans la conception et l'évaluation de nouvelles méthodes pour favoriser le maintien des personnes âgées à domicile. De telles collaborations sont nécessaires pour s'assurer d'un usage raisonnable et critique de nouvelles technologies.

\section{DÉFIS DES NOUVELLES TECHNOLOGIES DE SANTÉ}

Ingénieurs et chercheurs ont pour défi de réduire les contraintes ressenties par les personnes âgées, par exemple en simplifiant l'utilisation de ces nouvelles technologies de santé, en améliorant les algorithmes permettant de prévenir et d'identifier à distance les besoins d'aides et de soins des patients, en réduisant les interventions et manipulations des soignants nécessaires au bon fonctionnement de ces technologies, et en les rendant plus ergonomiques (taille, poids et confort).

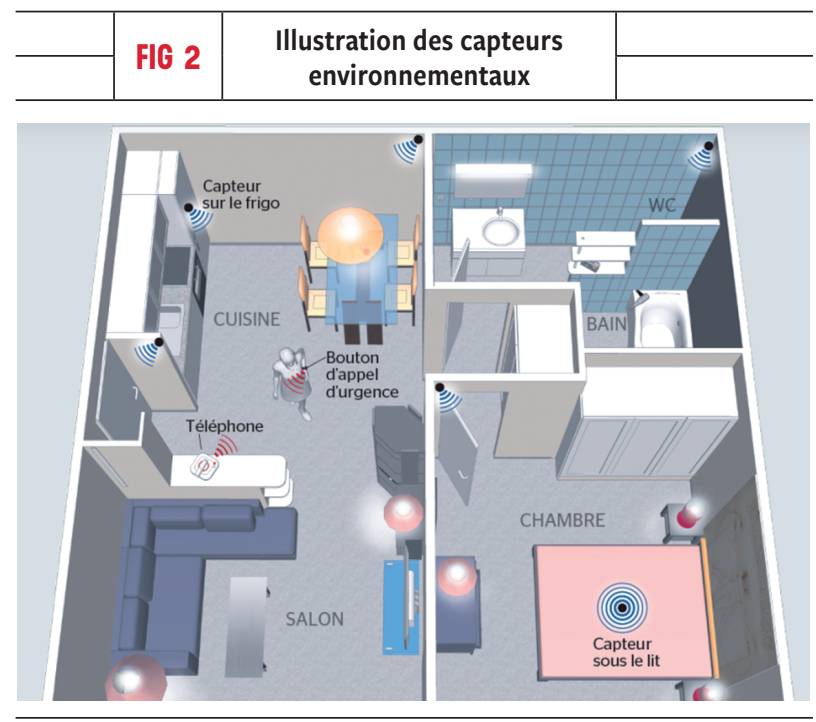

(Avec l'autorisation de J. Kummer, journaliste 24 Heures). 
L'utilisation de ces nouvelles technologies dans la pratique clinique relève aussi une dimension éthique qui souligne l'importance de respecter la dignité, l'intégrité et la sécurité du patient, ainsi que la confidentialité des données mesurées et enregistrées. ${ }^{23,24}$ Par exemple, dans le cadre du projet SWISKO, afin de respecter les exigences éthiques, une information orale et écrite sur les objectifs et le déroulement de l'étude a été transmise par un professionnel de santé aux patients potentiellement éligibles lors d'une visite à domicile. Afin d'être complets dans l'information transmise, les capteurs utilisés durant l'étude ont été présentés aux potentiels participants et les éventuelles questions du patient et de la famille ont été éclaircies. Finalement, la personne âgée acceptant de participer a signé une déclaration de consentement détaillant les contours de ses droits et devoirs, et précisant que ses données personnelles et médicales seront enregistrées sous une forme codée et consultées exclusivement par un nombre limité de personnes afin de pouvoir accomplir les tâches nécessaires au bon déroulement du projet. Toutes les données permettant d'identifier le patient (par exemple, le nom, la date de naissance) sont ainsi remplacées par un code.

\section{CONCLUSION}

Les nouvelles technologies de santé connectée peuvent apporter une solution pour soutenir le patient, les professionnels de santé, la famille et les proches-aidants souvent surchargés. Le but n'est pas de substituer le personnel soignant par la technologie, mais de développer un nouvel outil de travail pour un meilleur suivi de la personne âgée, en lui prodiguant des soins de qualité en toute sécurité, confort et liberté. Il s'agit également d'offrir, à l'aide des nouvelles technologies de la santé connectée, une meilleure qualité de vie aux personnes âgées vivant à domicile, en stimulant leur autonomie et en réduisant les risques d'hospitalisation. Les nouvelles technologies de la santé connectée, par un partage électronique et sécurisé des informations entre les professionnels de santé, le patient et les milieux de soins, sont un outil pertinent pour favoriser une prise en charge interprofessionnelle qui est une composante essentielle pour améliorer la qualité, la continuité des soins et la sécurité des patients.
Introduire des nouvelles technologies dans les systèmes de santé nécessite une coordination et une communication entre les différents professionnels de santé, les milieux de soins, les patients et les familles, ainsi qu'une prise en considération de la complexité d'implémenter en pratique clinique des projets technologiques..$^{25} \mathrm{Il}$ faut aussi évaluer scientifiquement l'utilité et l'impact de ces nouvelles technologies en pratique clinique, en s'assurant en particulier de respecter la dignité, l'intégrité et la sécurité du patient ainsi que la protection des données.

Remerciements: Nous remercions vivement NOMAD, les soins à domicile du canton de Neuchâtel, et plus particulièrement Mme Reynaud Senes et Mme Vrolixs, les responsables d'équipe et les infirmières et assistantes en soins et en santé communautaire, pour leur soutien et leur contribution au projet SWISKO. Nous remercions également Idiap Research Institute et l'équipe «Social Computing», et plus particulièrement Oya Aran, Dayra Sanchez-Cortes et Rui Hu pour leur travail dans le développement des algorithmes dans le projet SWISKO. Nous remercions CTI/Innosuisse pour leur soutien financier au projet SWISKO.

Conflit d'intérêts : Dr Philipp Buluschek est employé de l'entreprise DomoSatey. Les autres auteurs n'ont déclaré aucun conflit d'intérêts en relation avec cet article.

\section{IMPLICATIONS PRATIQUES}

- Face au vieillissement de la population, les nouvelles technologies de santé connectée peuvent offrir un potentiel intéressant pour améliorer la gestion des maladies chroniques et pour répondre au souhait des personnes âgées de vivre le plus longtemps possible à domicile

- Pour répondre au mieux aux besoins des personnes âgées, il est important de développer et tester les nouveaux outils technologiques en collaboration avec les patients, la famille et les différents professionnels de santé

- Introduire des nouvelles technologies dans les systèmes de santé nécessite une coordination et une communication entre les différents professionnels de santé, les milieux de soins, les patients et les familles, ainsi qu'une prise en considération de la complexité d'implémenter en pratique des projets technologiques, en s'assurant en particulier de respecter la dignité, l'intégrité et la sécurité du patient ainsi que la protection des données

\author{
1 Kucera J, Krummenacher A. La \\ population de la Suisse 2016. Office \\ fédéral de la statistique, 2017:349-1600. \\ (Pyramide des âges selon Scénario \\ A-00-2015 'référence': www.media-stat. \\ admin.ch/animated/chart/01 pyramid/ga-q- \\ 01.03.02-dashboard.html) \\ 2 World Health Organization, Depart- \\ ment of Economic and Social Affairs, \\ Population Division. World Population \\ Ageing 2013. ST/ESA/SER.A/348. \\ 3 Christensen K, Doblhammer G, Rau R, \\ et al. Ageing populations: the challenges \\ ahead. Lancet 2009;374:1196-208. \\ 4 Wieser S, Riguzzi M, Pletscher M, et al. \\ How much does the treatment of each \\ major disease cost? A decomposition of \\ Swiss National Health Accounts. Eur J \\ Health Econ 2018;19:1149-61. \\ 5 Füglister-Dousse S, Dutoit, Pellegrini S. \\ Soins de longue durée aux personnes \\ âgées en Suisse. Evolutions 2006-2013 \\ (Obsan Rapport 67). Neuchâtel:
}

Observatoire suisse de la santé, 2015. 6 Weber D, Abel B, Ackermann G, et al. Santé et qualité de vie des personnes âgées. Bases pour les programmes d'action cantonaux. Promotion Santé Suisse, Rapport 5, Berne et Lausanne, 2016.

7 Eng T. The e-Health Landscape: a terrain map of emerging information and communication technologies in health and health care. Princeton,2001, NJ: The Robert Wood Johnson Foundation. 8 World Health Organization, 2016. www.who.int/topics/ehealth/en/

$9{ }^{* *}$ Boulos MN, Lou RC, Anastasiou A, et al. Connectivity for healthcare and well-being management: examples from six European projects. Int J Environ Res Public Health 2009;6:1947-71.

10 * Patel S, Park H, Bonato P, et al. A review of wearable sensors and systems with application in rehabilitation. J Neuroeng Rehabil 2012;9:21.

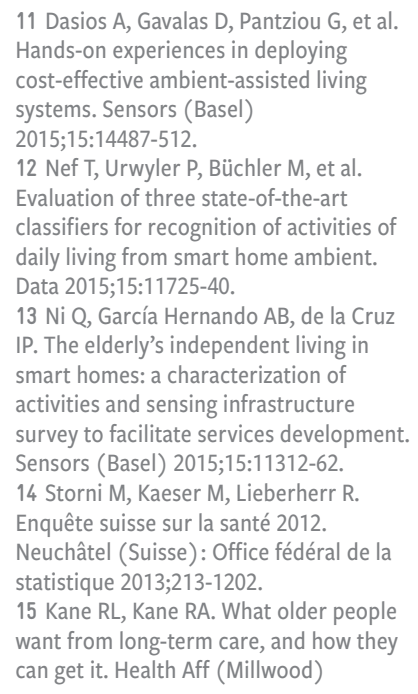

11 Dasios A, Gavalas D, Pantziou G, et al. Hands-on experiences in deploying cost-effective ambient-assisted living systems. Sensors (Basel)

2015;15:14487-512.

12 Nef T, Urwyler P, Büchler M, et al. Evaluation of three state-of-the-art classifiers for recognition of activities of daily living from smart home ambient. Data 2015;15:11725-40.

13 Ni Q, García Hernando AB, de la Cruz IP. The elderly's independent living in smart homes: a characterization of activities and sensing infrastructure survey to facilitate services development. Sensors (Basel) 2015;15:11312-62. 14 Storni M, Kaeser M, Lieberherr R. Enquête suisse sur la santé 2012.

Neuchâtel (Suisse): Office fédéral de la statistique 2013;213-1202.

15 Kane RL, Kane RA. What older people want from long-term care, and how they can get it. Health Aff (Millwood)

2001;20:114-27.

16 Oswald F, Wahl HW, Schilling O, et al. Relationships between housing and healthy aging in very old age. Gerontologist 2007; 47:96-107.

17 Meiland F, Innes A, Mountain G, et al. Technologies to support communitydwelling persons withvdementia: a position paper on issues regarding development, usability, effectiveness and cost-effectiveness, deployment, and ethics. JMIR Rehabil Assist Technol 2017;4:e1.

18 Mortenson WB, Sixsmith A, Beringer R. No place like home? Surveillance and what home means in old age. Can J Aging 2016;35:103-14.

19 Rialle V, Rumeau P, Ollivet C, et al. Télémédecine et gérontechnologie pour la maladie d'Alzheimer: nécessité d'un pilotage international par l'éthique. J Int Bioéthique 2014;25:127-45.

20 Peek ST, Wouters EJ, van Hoof J, et al. 
Factors influencing acceptance of technology for aging in place: a systematic review. Int J Med Inform

2014;83:235-48

21 Siegel C, Hochgatterer A, Dorner TE. Contributions of ambient assisted living for health and quality of life in the elderly and care services-a qualitative analysis from the experts' perspective of care service professionals. BMC Geriatr 2014;14:112.

22 *Peetoom KK, Lexis MA, Joore M, et al. Literature review on monitoring technologies and their outcomes in independently living elderly people. Disabil Rehabil Assist Technol

2014;10:271-94.

23 Aloulou H, Mokhtari M, Tiberghien T, et al. Deployment of assistive living technology in a nursing home environment: methods and lessons learned. BMC Med Inform Decis Mak 2013;13:42.

24 Pol M, van Nes F, van Hartingsveldt M, et al. Older people's perspectives

regarding the use of sensor monitoring in their home. Gerontologist 2016;56:485-93. $25 *$ Greenhalgh T. How to improve success of technology projects in health and social care. Public Health Res Pract 2018;28:e2831815.

* à lire
** à lire absolument 